\title{
Depression in Primary care: Interpersonal Counseling vs Selective serotonin reuptake inhibitors. The DEPICS Study. A multicenter randomized controlled trial. Rationale and design
}

Marco Menchetti ${ }^{1 *}$, Biancamaria Bortolotti ${ }^{1}$, Paola Rucci ${ }^{2,3}$, Paolo Scocco ${ }^{4,5}$, Annarosa Bombi ${ }^{1}$, Domenico Berardi ${ }^{1}$, DEPICS Study Group

\begin{abstract}
Background: Depression is a frequently observed and disabling condition in primary care, mainly treated by Primary Care Physicians with antidepressant drugs. Psychological interventions are recommended as first-line treatment by the most authoritative international guidelines but few evidences are available on their efficacy and effectiveness for mild depression.

Methods/Design: This multi-center randomized controlled trial was conducted in 9 Italian centres with the aim to compare the efficacy of Inter-Personal Counseling, a brief structured psychological intervention, to that of Selective Serotonin Reuptake Inhibitors. Patients with depressive symptoms referred by Primary Care Physicians to psychiatric consultation-liaison services were eligible for the study if they met the DSM-IV criteria for major depression, had a score $\geq 13$ on the 21-item Hamilton Depression Rating Scale, and were at their first or second depressive episode. The primary outcome was remission of depressive symptoms at 2-months, defined as a HDRS score $\leq 7$. Secondary outcome measures were improvement in global functioning and recurrence of depressive symptoms at 12-months. Patients who did not respond to Inter-Personal Counseling or Selective Serotonin Reuptake Inhibitors at 2-months received augmentation with the other treatment.
\end{abstract}

Discussion: This trial addresses some of the shortcomings of existing trials targeting major depression in primary care by evaluating the comparative efficacy of a brief psychological intervention that could be easily disseminated, by including a sample of patients with mild/moderate depression and by using different outcome measures.

Trial registration: Australian New Zealand Clinical Trials Registry ACTRN12608000479303

\section{Background}

Major Depression (MD) is an important public health problem, associated with high levels of disability, impairment in quality of life, and increased mortality rates $[1,2]$, similarly to severe medical conditions like congestive heart failure and diabetes [3]. Moreover, depression is associated with high health services utilization, work absenteeism, and decreased performances at work $[4,5]$ with elevated direct and indirect social costs. Epidemiological studies

\footnotetext{
*Correspondence: marco.menchetti3@unibo.it

1 Institute of Psychiatry, Bologna University, Bologna, Italy

Full list of author information is available at the end of the article
}

showed that MD has a high prevalence in primary care ranging from $2.6 \%$ to $29.5 \%$ [6-8].

Many patients with depression seek help in primary care and an increasing proportion has been treated in this setting, especially since the availability of safe and easy to use antidepressants (ADs) [9]. However, some problems remain in the management of depression in primary care, including the insufficient duration of antidepressant treatment and the limited use of non-pharmacological options [10-12]. In particular, psychological interventions are rarely used even for those patients who could benefit from them: patients suffering from mild depression related to stressful life events and 
patients in which the risk/benefits ratio of antidepressants is less favourable (e.g. elderly, frail patients, patients with polypharmacotherapy, women with postpartum depression). Several reasons account for this limited use: the small number of trained therapists in primary care $[13,14]$, the physician's positive opinion and attitude on drug treatment $[15,16]$, the few evidence on the comparative efficacy and effectiveness of brief psychotherapies vs antidepressants.

However, psychological interventions are often preferred by primary care patients $[17,18]$ and are recommended as first-line treatment by the most authoritative international guidelines $[19,20]$.The APA guidelines [19] stated that antidepressants or an effective psychotherapy alone may be considered as the first-line treatment for patients with mild to moderate major depression. The National Institute for Clinical Evidence (NICE) guidelines [20] recommend not to use antidepressants routinely to treat mild depression because the risk-benefit ratio is poor and suggest as an alternative a range of low-intensity psychosocial interventions.

These recommendations are based on few trials directly comparing antidepressant drugs with different kinds of psychological interventions in primary care [21-28]. Therefore the NICE guidelines underscore that adequately powered Randomized Controlled Trial (RCT) are warranted with representative participant samples, reporting relevant outcomes to assess the efficacy of psychological interventions and antidepressants [20].

The objectives of the RCT described in this paper are: 1) to evaluate the efficacy of a psychological intervention, the Inter-Personal Counseling (IPC), compared with Selective Serotonin Reuptake Inhibitors (SSRIs), for mild to moderate MD; 2) and to assess the efficacy of treatment augmentation with SSRI or IPC in patients who do not respond to monotherapy.

\section{Methods/Design \\ Study procedures Study design}

This multi-center randomized controlled trial was conducted in nine academic centres located in Northern, Central and Southern Italy: Bologna (coordinating centre), Bari, Cagliari, Foggia, Modena, Pavia, Perugia, Torino, Varese. The recruitment phase started on May 1st 2006 and finished on May 1st 2008. Each centre set up a specific collaborative programme with primary care physicians working in its area, with the aim to improve the management of depression. In some research units a collaborative programme was already in place at study inception, in others it was implemented on the occasion of the research. Primary Care Physicians (PCPs) were informed about the DEPICS study protocol during specific meetings and informal contacts; further, written materials about eligibility criteria and treatment algorithm were delivered.

Patients were recruited from university-based psychiatric consultation-liaison services specifically dedicated to PCPs. PCPs were encouraged to refer patients recognized as suffering from depressive symptoms; patients were seen by a consultant psychiatrist and evaluated for the possible inclusion in the study.

\section{Study protocol approval and trial registration}

Participation in the study was voluntary and written informed consent was obtained. Patients were informed that they could withdraw their consent to participate at any time, with no negative consequences on their future medical treatment. Patients who wished to withdraw from the study received care as usual. The study protocol was approved by the Ethical Committee of Azienda Ospedaliera Universitaria di Bologna. Eligible patients received an explanation of the study procedures and were asked to sign an informed consent. Written documentation about study methodology and procedures and a letter for PCP were delivered to all recruited patients. The PCPs were also periodically informed by letter or by phone about the course of illness of their patients during the study.

The trial was registered in the Australian New Zealand Clinical Trials Registry (ANZCTR) as ACTRN 12608000479303.

\section{Inclusion and exclusion criteria}

Adult patients suffering from depressive symptoms and referred from their PCP were potential candidates for the trial. Patients aged 18 years or older were eligible if they met criteria for a MD Episode, had a score $\geq 13$ on Hamilton Depression Rating Scale (HDRS, 21-item version) [29], and were at their first or second MD Episode treated with antidepressants or psychotherapy. Patients who met all inclusion/exclusion criteria but had a HDRS score $<13$ were reassessed after 1 month (watchful waiting arm); if their HDRS score was $\geq 13$ after 1 month they were enrolled and randomised to IPC or SSRI. Inclusion and exclusion criteria are shown in Table 1.

We chose to exclude patients with two or more previous depressive episodes treated with antidepressants or psychotherapy, with Borderline or Antisocial Personality Disorder because of the different pattern of response to treatment and the less favourable prognosis $[30,31]$.

After a baseline assessment, eligible patients were randomly allocated to a brief structured psychological intervention, IPC, or to antidepressant treatment with SSRI, the most used class of drugs in primary care.

\section{Randomization and treatment allocation}

Randomization sequences, derived from a computer random number generator, were delivered to each 
Table 1 Study recruitment: inclusion and exclusion criteria

\begin{tabular}{ll}
\hline Inclusion criteria & Exclusion criteria \\
\hline Age $\geq 18$ years & Current moderate to high suicide risk \\
DSM-IV Major Depressive Episode (MDE) & Current/past episodes of mania or hypomania \\
HDRS score $\geq 13$ & Current/past psychotic symptoms \\
First or second lifetime treated MDE ${ }^{\circ}$ & Borderline personality disorders \\
Availability to sign informed consent & Anti-social personality disorders \\
& Substance abuse or dependence \\
& Cognitive impairment \\
& Effective ongoing antidepressant treatment* \\
& Effective ongoing psychotherapy \\
& Poor knowledge of Italian language \\
& Pregnancy or breastfeeding
\end{tabular}

${ }^{\circ}$ Only previous episodes treated with specific therapeutic options (antidepressants, psychotherapy) were considered.

* Including antidepressants, mood stabilizers, and antipsychotics; only use of moderate dose of sedatives-hypnotics was allowed (doses $\leq 7.5$ mg lorazepam equivalent/day).

centre by the coordinating centre. In each centre, allocation to treatment group was made by dedicated research personnel outside the consultation-liaison service where patients were recruited, assessed and treated. After baseline assessment and after consent to participation in the study was obtained, the researcher was contacted via telephone by clinicians and disclosed the assignment.

\section{Blinding}

Raters who administered assessment's instruments were different from the clinicians who provided psychiatric consultation to PCPs and delivered pharmacological or psychological interventions. Efforts were made to keep raters blind to randomization assignment.

\section{Outcomes}

The primary outcome was the remission of depressive symptoms at the 2-month follow up visit, defined as a HDRS score of 7 or less. Secondary outcome measures were: improvement in global functioning and recurrence of depressive symptoms at the 12-months follow up visit. Patients with a HDRS score $\geq 13$ at 2 -month follow-up received augmentation with other treatment (Figure 1).

\section{Intervention}

\section{Interpersonal Counseling (IPC)}

IPC is a brief manualized psychological intervention, derived from Interpersonal Psychotherapy (IPT) and suitable for different medical contexts. This intervention can be delivered by trained mental health professionals, nurses and social workers [32-35]. IPC has been adapted and tested on patients with several conditions, in association with pharmacological treatment or alone. These conditions include subthreshold depression [36], major depression [37], depressive symptomatology after miscarriage [38], psychological symptoms after a major physical trauma [39], stress and distress without serious medical or psychiatric conditions [32], breast cancer [40], and distress after myocardial infarction [41].

IPC is focused on patients' current psychological problems and social functioning and specifically on four interpersonal problem areas: prolonged grief, interpersonal disputes, role transitions and interpersonal deficits. Patients are helped to identify effective strategies in order to deal with their interpersonal problems. In the original manual [33], IPC consisted of six thirty-minute sessions, with the initial session being longer (1 hour), and was self-dosing: the patient determined the number of sessions and many patients were satisfied with fewer than six sessions. In our study IPC has been adapted to accommodate patients' needs. The recommended number of therapy sessions was six thirty-minute weekly sessions. Therapists determined if one or two additional sessions were needed $(6+1$ or $6+2)$. IPC sessions were videotaped if patients provided a written informed consent.

Training of IPC therapists IPC was delivered by 18 therapists (Bologna 3, Modena 2, Torino 3, Pavia 2, Varese 2, Perugia 1, Foggia 3, Bari 1, Cagliari 1). Therapists were residents in psychiatry or in clinical psychology with a clinical experience of at least 2 years. They attended a 3-day teaching seminar on interpersonal theory's foundations and IPC structure and techniques conducted by one of the authors (PS), who had previously trained and supervised the Italian IP-therapists of a cross-national RCT $[42,43]$. Before starting the formal training, trainees were asked to read the Italian translation of the IPT manual [44] and the IPC treatment manual by Weissman [30]. During the teaching seminars, videotapes of psychotherapy sessions were presented and discussed, before simulating an IPC session with a role-playing. The candidates were encouraged to use the interpersonal approach when treating their patients (out of the trial). 


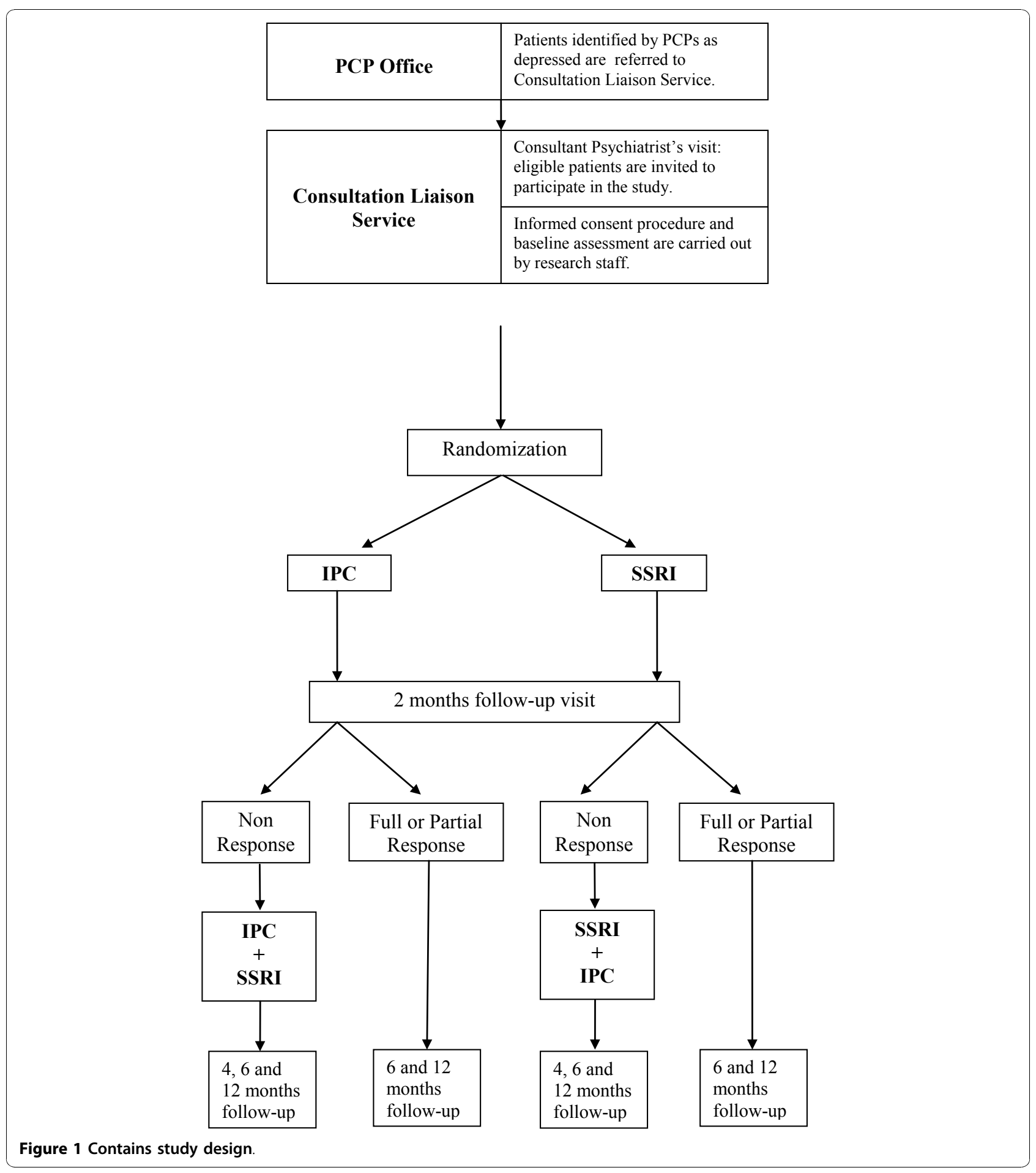

IPC group supervision Subsequently, monthly group supervisions were scheduled with one of the authors (PS) in order to discuss cases, to ensure the intervention's consistency. At each meeting the trainees reported the problems and challenges they experienced with IPC. Trainees were requested to present four or five IPC videotaped sessions to review during group supervision, choosing the more challenging sessions. These videotapes provided indications on the communication style, techniques and strategies applied by therapists. In this way, during the supervision meeting, trainees could learn from the supervisor, but also vicariously from one 
another (peer-supervision). Working with a group of trainees means that each could be learning even while one is the particular focus of the supervisor attention. The supervisor stimulated trainees to use a peersupervision method with all the participants actively involved in the case discussion with suggestions or comments. As Hillerbrand [45] pointed out, group members are often able to give feedback to one another that is more understandable than what the supervisor offers. Then, peer-supervision was used in any unit between the monthly-group supervisions.

\section{Antidepressant treatment}

Because our aim was to assess the antidepressant treatment for depression as delivered routinely in clinical practice, we chose two antidepressants, sertraline and citalopram, among the available SSRIs. These agents have minimal or modest effects on the cytochrome P-450 isoenzyme system which makes them safe even in patients with physical illnesses and taking other medications. Moreover, sertraline and citalopram had the lowest cost among SSRI antidepressants in Italy at study start.

Citalopram was started at $10-20 \mathrm{mg}$ and titrated if needed to $60 \mathrm{mg}$ and sertraline was started at $25-50 \mathrm{mg}$ and titrated up to $200 \mathrm{mg}$. The pharmacological treatment was continued for at least 4-6 months after the patient had responded as suggested by international guidelines $[19,20]$. The psychiatrist was free to choose between the two pharmacological options according to his/her personal preference and informed the PCP by letter about the suggested pharmacological treatment. Moreover he/she provided psycho-education about antidepressants and their side effects. Two or three subsequent visits with the consultant psychiatrist were planned every 2-3 weeks, lasting around 15 minutes in order to evaluate patients' compliance, clinical response and side effects; a specific form was used to assess adverse events. The only treatment modification allowed was the switch from sertraline to citalopram and vice versa.

There were no limitations on the number of PCP's visits. Concurrent use of sedatives-hypnotics was allowed up to a dose $<7.5 \mathrm{mg}$ lorazepam equivalent/day in both treatment arms. During the study, other psychotropic medications were forbidden; if needed, the patient was terminated from the protocol and referred to his or her PCP for alternative treatments.

\section{Combined treatment}

Patients with a HDRS score $\geq 13$ at 2-months follow-up received augmentation with other treatment. In particular those in the SSRI arm began a regular 6-sessions IPC; those in IPC arm began the antidepressant therapy and continued IPC for further 4-6 sessions.

\section{Assessments}

At baseline, patients filled out socio-demographic and clinical forms, including information about medical history, current pharmacological treatments and health services utilization in the last 6 months (including information about use of both general health and specialist care and about laboratory tests, instrumental procedures, admission to hospital). Follow-up visits were scheduled at 2, 4, 6 months and 1 year; the 4-month assessment was scheduled only for non-responders who started the combined treatment at 2 months. Table 2 summarizes instruments administered at each visits.

The assessment was conducted by interviewers not involved in patients treatment and trained to the use of instruments and scales. Research personnel was trained to the use of study instruments in 2 investigator's meetings, in which videotaped interviews were presented and rated. In particular, a structured interview guide for the HDRS was employed in order to facilitate the achievement of good inter-rater reliability [46]. Certification in the use of the HDRS was obtained when the total score did not differ by more than 4 points with the gold standard (MM) on 3 videotaped cases.

\section{Diagnostic assessment}

The diagnostic assessment was carried out at baseline using the Mini International Neuropsychiatry Interview (MINI) Plus [47], an instrument that allows to make a diagnosis according to DSM-IV or International Classification of Diseases (ICD-10) criteria. The reliability, sensitivity, and specificity of the MINI are equivalent to those of the Composite International Diagnostic Interview (CIDI) [48] and the Structured Clinical Interview for DSM-IV [47] in a clinical population.

The assessment was completed with the section on diagnosis of borderline personality disorders of the SCID-II; we used the self-report scale followed, if necessary, by a semi-structured interview [49].

\section{Depressive symptoms}

Severity of depressive symptoms was assessed using the 21-item Hamilton Rating Scale for Depression (HDRS21) [29] the most widely used outcome measure in trials on depression. A HDRS score $<=7$ denotes the absence of depression, from 8 to 17 mild depression, from 18 to 24 moderate depression and a score $\geq 25$ indicates severe depression [50]. In patients aged 65 years or more depressive symptom severity was also evaluated using the Geriatric Depression Scale (GDS) [51].

\section{Functioning}

We used the Work and Social Adjustment Scale (WSAS), a self-report 5-item scale that measures functional impairment attributable to an identified problem 
Table 2 Assessment instruments at each time point

\begin{tabular}{|c|c|c|c|c|c|}
\hline Instrument & Baseline & & low-up visits & & \\
\hline & & 2 months & 4 months & 6 months & 12 months \\
\hline MINI Plus & $x$ & & & & $x$ \\
\hline SCID ॥ & $x$ & & & & \\
\hline HDRS-21 & $x$ & $x$ & $x$ & $x$ & $x$ \\
\hline GDS (only patients aged 65 or more) & $x$ & $x$ & $x$ & $x$ & $x$ \\
\hline WSAS & $x$ & $x$ & $x$ & $x$ & $x$ \\
\hline WHOQOL BREF & $x$ & $x$ & $x$ & $x$ & $x$ \\
\hline |IP-64 & $x$ & & & & $x$ \\
\hline $\mathrm{IPQ}$ & $x$ & $x$ & & & $x$ \\
\hline ASQ & $x$ & & & & \\
\hline GSQ & & $x$ & & $x$ & $x$ \\
\hline
\end{tabular}

List of abbreviations: ASQ = Attachment Style Questionnaire, GDS = Geriatric Depression Scale, GSQ = General Satisfaction Questionnaire, HDRS = Hamilton Depression Rating Scale, IPQ = Interpersonal Problem Questionnaire, IIP-64 = 64-item Inventory of Interpersonal Problems, MINI Plus = Mini International Neuropsychiatry Interview Plus, SCID II = Structured Clinical Interview for DSM-IV Axis II Personality Disorders, WSAS = Work and Social Adjustment Scale, WHOQOL $=$ World Health Organization Quality Of Life.

or disorder. WSAS items investigate ability to work, home management, social leisure, private leisure, and relationships. A score from 11 to 20 denotes mild disability, while a score higher than 20 denotes severe disability [52].

\section{Quality of life}

The World Health Organization Quality Of Life (WHOQOL) - BREF is used to assess quality of life [53]. It consists of 24 items organized into four domains: physical health, psychological health, social relationships, and environment. Two additional items measure the overall perception of quality of life and health. Domain scores are scaled in a positive direction: higher scores denote higher quality of life [54].

\section{Interpersonal areas}

We used two instruments:

1) Interpersonal Problems Questionnaire (IPQ) is a brief self-report assessment measure, which queries life events that are relevant to the four focal interpersonal problem areas addressed in IPT: unresolved grief, role transitions, interpersonal role disputes and interpersonal deficits. [55]. It includes 3 different sections, the first investigating interpersonal relationships, the second focused on social life and close relationships and the last exploring the presence of significant life events occurred in the past 12 months.

2) The 64-item Inventory of Interpersonal Problems (IIP-64) is self-report measure of maladaptive relationship behaviour used to identify dysfunctional patterns in interpersonal interactions. This inventory assesses the severity of interpersonal problems in 8 domains: domineering or controlling, vindictive or self-centred, cold and distant, socially inhibited, non-assertive, overly accommodating, self-sacrificing, and intrusive or needy.
The IIP-64 has been widely used to assess psychotherapy [56].

\section{Other instruments used in the study}

In patients aged 65 years or more, if cognitive impairment was suspected, the Mini Mental State Examination (MMSE) was administered at baseline [57]; patients with a score of 27 or less were excluded from the study.

Moreover, we used the Attachment Style Questionnaire (ASQ), a 40-item self-report Likert Scale specifically designed to assess the type of attachment to significant other. The ASQ includes five scales: confidence (in self and others), discomfort with closeness, need for approval, preoccupation with relationships, and relationship as secondary (to achievement). Each subscale represents a dimension central to adult attachment and each item is scored on a 6-point scale ranging from totally agree to totally disagree [58].

Patient's satisfaction was measured only at the follow up visits with the General Satisfaction Questionnaire (GSQ), a self-report 7-item assessment scale that measures satisfaction with treatment received and with health services accessibility and acceptability [59].

\section{Data analysis}

\section{Data management and study monitoring}

Data were recorded by research assistants in a database developed in MS-ACCESS. The data quality was checked on a regular basis to ensure that data analysis could be promptly conducted.

\section{Sample size}

The sample size was determined using remission measured with the HDRS as the primary outcome. Data from previous RCT on MD showed that the remission rate with SSRI treatment is 35\%[60]. Other studies conducted in primary care reported higher remission rates 
with SSRI, ranging from 52 to $67 \%$ [24,61]. Assuming an intermediate $45 \%$ remission rate with SSRI and a clinically significant difference of $15 \%$ between the two treatments, a sample size of 274 (137 per arm) was determined that would result in a power of $80 \%$ at 0.05 alpha level. Considering a drop-out rate of about $10 \%$ at the first 2 months follow up, we planned to enrol 300 patients (150 per arm).

\section{Statistical analysis}

The statistical analysis plan includes the use of logistic regression analysis to model the probability of remission at 2 and 6 months as a function of randomization assignment. For this analysis, patients dropped out from the study are considered as non-remitters. The same model is used to predict the need of combined treatment (coded as yes/no) as a function of patient demographic and clinical characteristics.

Linear mixed models are used to analyse functional change and change in quality of life from baseline as a function of treatment, using the baseline score as a covariate. Satisfaction with treatment is compared between the treatment arms using Mann-Whitney test.

\section{Discussion}

Many studies have been conducted on the treatment of depression, but mostly on patients with moderate to severe forms in the mental health setting. Relatively few trials have been carried out with patients with mild depression in primary care. In particular, evidence on the efficacy of psychological interventions in comparison with antidepressants is scanty. To our knowledge only eight RCTs comparing different psychological interventions and antidepressants were conducted in primary care [21-28]. However, methodological issues limit the findings of these trials: first, they had very small sample sizes, thereby increasing the likelihood of Type II error; moreover the three older ones used tricyclic antidepressants that are now rarely prescribed [21-23]; finally, two studies recruited only patients suffering from minor depression or dysthimia $[27,28]$. As suggested by several authors, it is crucial that RCTs on psychological interventions conducted in primary care include representative participant samples, use multiple outcome measures, test interventions easy to disseminate and evaluate differences in treatment response as a function of co-existent psychiatric problems and other medical problems [13,62-64]. Considering the challenges researchers must overcome, the DEPICS study has some points of strengths:

1) The use of broad eligibility criteria in order to obtain a representative sample, including patients with mild depression and depressive disorders comorbid with anxiety disorders or physical illnesses, very common in primary care setting.

2) The use of different outcome measures and a broad assessment including severity of depressive symptom, functioning, quality of life, interpersonal relationships, and patient's satisfaction,

3) The evaluation of the efficacy of a brief structured psychological intervention (IPC) as a monotherapy for $\mathrm{MD}$ in primary care.

Among evidence-based psychotherapies for depression, the interpersonal approach is one of the most supported, but qualified psychotherapists are often not available in primary care. If we want to see widespread implementation of effective practices such as evidence -based psychotherapies, we need to develop and test "simpler" versions of evidence based psychosocial treatment that could be provided by different health-care professionals who do not necessarily have mental health training [64]. With a view of moving from research evidence to practice, IPC lends itself to be disseminated in primary care, because it is a briefer version of evidence-based psychosocial treatments and could be provided by clinicians with less mental health expertise than IPT or CBT therapists. The efficacy of IPC has been previously tested in patients with depressive symptoms related to current stress (mainly physical illnesses) and subthreshold depression or in individual, group and telephone format $[32,37-41,65]$. One study evaluated the efficacy of IPC plus venlafaxine for MD in primary care [36]. Moreover, Bolton et al [65] tested the efficacy of group IPC in alleviating depressive symptoms and dysfunction in patients with sub-threshold or major depression in Uganda. However, IPC was not previously tested as an individual intervention in monotherapy for patients with MD in primary care.

An important limitation of this study is the absence of a placebo group, usually characterized by a high rate of spontaneous remission (27\% to $43 \%)[22,66,67]$. However, the present study is part of a more comprehensive collaborative programme between primary care and mental health services and we chose to employ only an active comparator in the protocol to match the real-world clinical setting as closely as possible. Another limitation is the exclusion of patients with more than one clinically significant depressive episode in their personal history. We chose to exclude these patients because of the different pattern of response to treatment and the less favourable prognosis [9]. Our results therefore cannot be generalized to patients with chronic or recurrent MD.

In conclusion, the present trial aims to provide evidence on the efficacy of a brief and easy to implement psychological intervention compared to the most frequently used drug treatments for major depression. 


\section{Acknowledgements}

We want to acknowledge the ideas and the support of professor D.

Goldberg, Institute of Psychiatry, King's College London.

Moreover we are grateful to F. Asioli for his suggestions in the protocol development and to R. N. Forgione for his assistance in database

development and management.

We wish to thank for their help, the members of Italian Society of General

Practice, P. Carbonatto and T. Scarponi.

We thank all the members of the DEPICS Study Group:

V. Affatati ${ }^{a}$, G. Alberini ${ }^{b}$, F. Baranzini ${ }^{b}$, S. Bellino ${ }^{c}$, A Bellomo $^{d}$, T. Blasi $^{e}$, F Bogetto $^{c}$, P. Bortolaso ${ }^{b},{ }^{\prime}$ C. Callegari ${ }^{b}$, B. Carpiniello ${ }^{f}, N^{\prime}$. Colombini ${ }^{g}, C^{\prime}$ ' Contu' G. Croci ${ }^{b}$, M. De Salvia ${ }^{d}$, M. Diurni ${ }^{b}$, S. Elisei ${ }^{e}$, M. Ferretti ${ }^{d}$, P. Fiore ${ }^{d}$, P. FusarPoli', S. Iuso ', A. Lacalamita ${ }^{\prime}$, T. La Ferla ${ }^{e}$, L. Lia', C.C. Luciano', M. Magnani', D. Manganaroi, V. Martinelli ${ }^{\text {h }}$, I. Martino ${ }^{\text {a }}$, M. B. Montaguti', C. Nespecai, A.

Petito $^{d}$, F. Pinna ${ }^{f}$, M. Pisellie, P. Politi ${ }^{h}$, R. Quartesan', A. Rella ${ }^{e}$, F. Restaino ${ }^{h}$, M. Rigatelli ${ }^{9}$, P. Sciarini ${ }^{h}$, E. Simoni ${ }^{9}$, M. Succu', E. Tedeschini ${ }^{9}$, O. Todarello ${ }^{\text {a }}$, S. Vender $^{\text {b }}$, L. Zaccagni ${ }^{\mathrm{e}}$, M. Zizza

a Department of Psychiatry, University of Bari, Bari, Italy.

b Department of Medicine, University of Insubria, Varese, Italy

c Unit of Psychiatry, Department of Neurosciences, University of Turin, Turin, Italy

d Section of Psychiatry and Clinical Psychology, Department of Medical Sciences, University of Foggia, Foggia, Italy

e Department of Clinical and Experimental Medicine, (Division of Psychiatry, Clinical Psychology and Psychiatric Rehabilitation), University of Perugia, Perugia, Italy

${ }^{f}$ Department of Mental Health, Section of Psychiatry, University of Cagliari, Cagliari, Italy.

${ }^{g}$ Mental Health Department, USL Modena, Italy

h Department of Health Applied Sciences, Section of Psychiatry, University of Pavia, Pavia, Italy

'Institute of Psychiatry, Bologna University, Bologna, Italy

We also thank G. Lullini, and E. Pedrini (Institute of Psychiatry, Bologna University), A. D'Onghia, M. Mazza, and S. Papagni (Section of Psychiatry and Clinical Psychology, Department of Medical Sciences, University of Foggia) for their collaboration to the study.

\section{Funding}

The study was funded by the Italian Ministry for Education, University and Research (Prot. 2005063749).

\section{Author details}

${ }^{1}$ Institute of Psychiatry, Bologna University, Bologna, Italy. ${ }^{2}$ Department of Medicine and Public Health, Bologna, Italy. ${ }^{3}$ Department of Psychiatry, University of Pittsburgh School of Medicine, Pittsburgh, PA, USA. ${ }^{4}$ Psychiatric Clinic, Department of Neuroscience, Padova University, Padova, Italy. ${ }^{5}$ Mental Health Department, ULSS 16 Padova, Padova, Italy.

\section{Authors' contributions}

$\mathrm{DB}, \mathrm{MM}, \mathrm{BB}$ conceived the study and developed the study protocol. DB is the principal investigator that assembled the group of investigators. MM and $\mathrm{BB}$ wrote the first draft of this manuscript, describing the trial protocol. PR provided statistical advice in the design of the study and its on-going evolution. PS, MM and BB participated in the design and in the planning of the interventions. PR, BB and $A B$ managed the trial data. All authors have read and corrected draft versions, and approved the final version.

\section{Competing interests}

The authors declare that they have no competing interests.

Received: 30 August 2010 Accepted: 25 November 2010 Published: 25 November 2010

\section{References}

1. Ustun TB, Ayuso-Mateos JL, Chatterji S, Mathers C, Murray CJ: Global burden of depressive disorders in the year 2000. Br J Psychiatry 2004, 184:386-392

2. Cuijpers P, Smit F: Excess mortality in depression: a meta-analysis of community studies. J Affect Disord 2002, 72(3):227-36.
3. Hays RD, Wells KB, Sherbourne CD, Rogers W, Spritzer K: Functioning and well-being outcomes of patients with depression compared with chronic general medical illnesses. Arch Gen Psychiatry 1995, 52:11-19.

4. Wang PS, Simon G, Kessler RC: The economic burden of depression and the cost-effectiveness of treatment. Int J Methods Psychiatr Res 2003, 12:22-33.

5. Lerner D, Adler DA, Chang H, Lapitsky L, Hood MY, Perissinotto C, et al: Unemployment, job retention, and productivity loss among employees with depression. Psychiatr Serv 2004, 55:1371-1378.

6. Goldberg D, Lecrubier Y: Form and frequency of mental disorders across centers. In Mental IIIness in General Health Care: an International Study. Edited by: Ustun T, Sartorius N. John Wiley 1995.

7. Berardi D, Leggieri G, Berti Ceroni F, et al: Depression in primary care. A nationwide epidemiological survey. Family Practice 2002, 19(4):397-400.

8. King M, Nazareth I, Levy G, Walker C, Morris R, Weich S, et al: Prevalence of common mental disorders in general practice attendees across Europe. Br J Psychiatry 2008, 192:362-367.

9. Goldberg D, Goodyear I: The origins and Course of Common Mental Disorders. Routledge; East Sussex; 2005.

10. Von Korff MR: Improving outcomes in depression. BMJ 2001, 323:948-949.

11. Robinson WD, Geske JA, Prest LA, Barnacle R: Depression treatment in primary care. J Am Board Fam Pract 2005, 18:79-86.

12. Martín-Agueda B, López-Muñoz F, Rubio G, Guerra JA, Silva A, Alamo C: Management of depression in primary care: a survey of general practitioners in Spain. Gen Hosp Psychiatry 2005, 27(5):305-12.

13. Wolf NJ, Hopko DR: Psychosocial and pharmacological interventions for depressed adults in primary care: a critical review. Clin Psychol Rev 2008, 28:131-161.

14. Layard R: The case for psychological treatment centres. BMJ 2006, 332(7548):1030-2

15. Williams JW Jr, Rost K, Dietrich AJ, Ciotti MC, Zyzanski SJ, Cornell J: Primary care physicians' approach to depressive disorders. Effects of physician specialty and practice structure. Arch Fam Med 1999, 8(1):58-67.

16. Figueiras A, Caamano F, Gestal-Otero JJ: Influence of physician's education, drug information and medical-care settings on the quality of drugs prescribed. Eur J Clin Pharmacol 2000, 56:747-753.

17. Dwight-Johnson M, Sherbourne CD, Liao D, Wells KB: Treatment preferences among depressed primary care patients. J Gen Intern Med 2000, 15:527-534.

18. van Schaik DJ, Klijn AF, van Hout HP, van Marwijk HW, Beekman AT, de Haan $M$, et al: Patients' preferences in the treatment of depressive disorder in primary care. Gen Hosp Psychiatry 2004, 26:184-189.

19. American Psychiatric Association: Guideline watch: practice guideline for the treatment of patients with major depressive disorder., 22005 [http:// www.psychiatryonline.com/pracGuide/pracGuideTopic 7.aspx].

20. National Institute for Health and Clinical Excellence: Depression: the Treatment and Management of Depression in Adults. 2009 [http://www. nice.org.uk/CG90], Published October 28.

21. Scott Al, Freeman CP: Edinburgh primary care depression study: treatment outcome, patient satisfaction, and cost after 16 weeks. BMJ 1992, 304(6831):883-7

22. Mynors-Wallis LM, Gath DH, Lloyd-Thomas AR, Tomlinson D: Randomised controlled trial comparing problem solving treatment with amitriptyline and placebo for major depression in primary care. BMJ 1995, 310:441-445.

23. Brown C, Schulberg HC, Madonia MJ, Shear MK, Houck PR: Treatment outcomes for primary care patients with major depression and lifetime anxiety disorders. Am J Psychiatry 1996, 153:1293-1300.

24. Mynors-Wallis LM, Gath DH, Day A, Baker F: Randomised controlled trial of problem solving treatment, antidepressant medication, and combined treatment for major depression in primary care. BMJ 320:26-30.

25. Bedi N, Chilvers C, Churchill R, Dewey M, Duggan C, Fielding K, et al: Assessing effectiveness of treatment of depression in primary care. Partially randomised preference trial. Br J Psychiatry 2000, 177:312-318.

26. Salminen JK, Karlsson H, Hietala J, Kajander J, Aalto S, Markkula J, et al: Short-term psychodynamic psychotherapy and fluoxetine in major depressive disorder: a randomized comparative study. Psychother Psychosom 2008, 77:351-357.

27. Williams JW, Barrett J, Oxman T, Frank E, Katon W, Sullivan M, et al: Treatment of dysthymia and minor depression in primary care: a randomized controlled trial in older adults. JAMA 2000, 284(12):1519-26. 
28. Barrett JE, Williams JW Jr, Oxman TE, Frank E, Katon W, Sullivan M, et al: Treatment of dysthymia and minor depression in primary care: a randomized trial in patients aged 18 to 59 years. J Fam Pract 2001, 50(5):405-12

29. Hamilton M: Development of a rating scale for primary depressive illness. Br J Soc Clin Psychol 1967, 6:278-296.

30. Vuorilehto MS, Melartin TK, Isometsä ET: Course and outcome of depressive disorders in primary care: a prospective 18-month study. Psychol Med 2009.

31. Brown C, Schulberg HC, Prigerson HG: Factors associated with symptomatic improvement and recovery from major depression in primary care patients. Gen Hosp Psychiatry 2000, 22(4):242-50.

32. Klerman GL, Budman S, Berwick D, Weissman MM, mico-White J, Demby A, et al: Efficacy of a brief psychosocial intervention for symptoms of stress and distress among patients in primary care. Med Care 1987, 25:1078-1088.

33. Weissman MM: Interpersonal counselling for stress and distress in primary care: a treatment manual. 1988 [http://mmw3@Columbia.edu]

34. Weissman MM, Markowitz JC, Klerman GL: Comprehensive guide to interpersonal psychotherapy. New York: Basic Books; 2000.

35. Judd F, Weissman M, Davis J, Hodgins G, Piterman L: Interpersonal counselling in general practice. Aust Fam Physician 2004, 33:332-337.

36. Mossey JM, Knott KA, Higgins M, Talerico K: Effectiveness of a psychosocial intervention, interpersonal counseling, for subdysthymic depression in medically ill elderly. J Gerontol A Biol Sci Med Sci 1996, 51: M172-M178

37. Judd FK, Piterman L, Cockram AM, McCall L, Weissman MM: A comparative study of venlafaxine with a focused education and psychotherapy program versus venlafaxine alone in the treatment of depression in general practice. Hum Psychopharmacol 2001, 16:423-428.

38. Neugebauer R, Kline J, Bleiberg K, Baxi L, Markowitz JC, Rosing M, et al: Preliminary open trial of interpersonal counseling for subsyndromal depression following miscarriage. Depress Anxiety 2007, 24:219-222.

39. Holmes A, Hodgins G, Adey S, Menzel S, Danne P, Kossmann T, et al: Trial of interpersonal counselling after major physical trauma. Aust N Z J Psychiatry 2007, 41:926-933.

40. Badger T, Segrin C, Dorros SM, Meek P, Lopez AM: Depression and anxiety in women with breast cancer and their partners. Nurs Res 2007, 56:44-53.

41. Oranta O, Luutonen S, Salokangas RK, Vahlberg T, Leino-Kilpi H: The outcomes of interpersonal counselling on depressive symptoms and distress after myocardial infarction. Nord J Psychiatry 2010, 64(2):78-86.

42. Frank E, Scocco P: IPT training and supervision in Italy: a model for the clinical psychiatrist across geographic and linguistic boundaries. Bulletin of the International Society for Interpersonal Psychotherapy 2003, 2(1):2-4.

43. Frank $E$, Cassano GB, Rucci $P$, Fagiolini A, Maggi L, Kraemer HC, et al: Addressing the Challenges of a Cross-National Investigation: Lessons from the Pittsburgh-Pisa Study of Treatment-Relevant Phenotypes of Unipolar Depression. Clinical Trial 2008, 5:252-261.

44. Klerman GL, Weissman MM, Rounsaville BJ, Chevron ES: Interpersonal Psychotherapy of Depression. New York: Basic Books; 1984.

45. Hillerbrand ET: Cognitive differences between expert and novices: implication for group supervision. J of Counseling and Development 1989, 67:293-296.

46. Williams JB: A structured interview guide for the Hamilton Depression Rating Scale. Arch Gen Psychiatry 1988, 45:742-747.

47. Sheehan DV, Lecrubier $Y$, Sheehan $\mathrm{KH}$, Amorim $\mathrm{P}$, Janavs J, Weiller $\mathrm{E}$, et al: The Mini-International Neuropsychiatric Interview (M.I.N.I.): the development and validation of a structured diagnostic psychiatric interview for DSM-IV and ICD-10. J Clin Psychiatry 1998, 59(Suppl 20):22-33.

48. Lecrubier $Y$, Sheehan DV, Weiller E, Amorim P, Bonora I, Sheehan KH, et al: The Mini Intenational Neuropsychiatric Interview (MINI). A short diagnostic structured interview: reliability and validity according to the CIDI. Eur Psychiatry 1997, 12(5):224-231.

49. First MB, Gibbon M, Spitzer RL, Williams J, Benjamin L: Structured Clinical Interview for DSM-IV Axis II Personality Disorders (SCID-II). American Psychiatric Press, Washington; 1998.

50. Moller $\mathrm{H}$ : Methodological aspects in the assessment of severity of depression by the Hamilton Depression Scale. Eur Arch Psychiatry Clin Neurosci 2001, 251(Suppl 2):I113-20.
51. Yesavage JA, Brink TL, Rose TL, Lum O, Huang V, Adey M, et al: Development and validation of a geriatric depression screening scale: a preliminary report. J Psychiatr Res 1982, 17:37-49.

52. Mundt JC, Marks IM, Shear MK, Greist JH: The Work and Social Adjustment Scale: a simple measure of impairment in functioning. Br J Psychiatry 2002, 180:461-464

53. De Girolamo G, Rucci P, Scocco P, Becchi A, Coppa F, D'Addario A, et al: Quality of life assessment: validation of the Italian version of the WHOQOL-Brief]. Epidemiol Psichiatr Soc 2000, 9:45-55.

54. Skevington SM, Lotfy M, O'Connell KA: The World Health Organization's WHOQOL-BREF quality of life assessment: psychometric properties and results of the international field trial. A report from the WHOQOL group. Qual Life Res 2004, 13:299-310.

55. Frank, Andrade : personal communication. 2003.

56. Horowitz, Alden, Wiggins, Pincus : Inventory of Interpersonal Problems (IIP-64). The Psychological Corporation, a Harcourt Assessment Company; 2000.

57. Folstein MF, Robins LN, Helzer JE: The Mini-Mental State Examination. Arch Gen Psychiatry 1983, 40:812

58. Feeney JA, Noller P, Hanrahan M: Assessing adult attachment: Developments in the conceptualization of security and insecurity.Edited by: Sperling MB, Berman WH. Attachment in adults: Theory, assessment and treatment New York: Guilford; 1994:128-152.

59. Huxley $P$, Warner R: Case management, quality of life, and satisfaction with services of long-term psychiatric patients. Hosp Community Psychiatry 1992, 43:799-802

60. Thase ME, Entsuah AR, Rudolph RL: Remission rates during treatment with venlafaxine or selective serotonin reuptake inhibitors. $\mathrm{Br} J$ Psychiatry 2001, 178:234-241.

61. McPartlin GM, Reynolds A, Anderson C: A comparison of once daily venlafaxine $X R$ and paroxetine in depressed outpatients treated in general practice. Primary Care Psychiatry 1998, 4:127-132

62. Scott J, Sensky T: Methodological aspects of randomized controlled trials of psychotherapy in primary care. Psychol Med 2003, 33:191-196.

63. Bortolotti B, Menchetti M, Bellini F, Montaguti MB, Berardi D: Psychological interventions for major depression in primary care: a meta-analytic review of randomized controlled trials. Gen Hosp Psychiatry 2008, 30(4):293-302.

64. Unützer J: Evidence-based treatments for anxiety and depression: lost in translation? Depress Anxiety 2008, 25(9):726-9.

65. Bolton P, Bass J, Neugebauer R, Verdeli H, Clougherty KF, Wickramaratne P, et al: Group interpersonal psychotherapy for depression in rural Uganda: a randomized controlled trial. JAMA 2003, 289:3117-3124.

66. Wade A, Michael LO, Bang HK: Escitalopram $10 \mathrm{mg} /$ day is effective and well tolerated in a placebo-controlled study in depression in primary care. Int Clin Psychopharmacol 2002, 17:95-102

67. Lepola UM, Loft $H$, Reines EH: Escitalopram (10-20 mg/day) is effective and well tolerated in a placebo-controlled study in depression in primary care. Int Clin Psychopharmacol 2003, 18:211-217.

Pre-publication history

The pre-publication history for this paper can be accessed here: http://www.biomedcentral.com/1471-244X/10/97/prepub

doi:10.1186/1471-244X-10-97

Cite this article as: Menchetti et al:: Depression in Primary care:

Interpersonal Counseling vs Selective serotonin reuptake inhibitors. The DEPICS Study. A multicenter randomized controlled trial. Rationale and design. BMC Psychiatry 2010 10:97. 